Appl i cat i on and val i dat i on of a new hi st ol ogi c st agi ng and grading system for pri mary bi I i ary ci rrhosi s

\begin{tabular}{|l|l|}
\hline 著者 & $\begin{array}{l}\text { Har ada Keni chi, Hsu Nayl ee, I keda Hi roko, } \\
\text { Zeni ya M ki o, Nakanuma Yasuni }\end{array}$ \\
\hline $\begin{array}{l}\text { j our nal or } \\
\text { publ i cat i on ti tl e }\end{array}$ & Journal of Cl i ni cal Gast r oent er ol ogy \\
\hline vol une & 47 \\
\hline number & 2 \\
\hline page range & 174 181 \\
\hline year & 2013 02-01 \\
\hline URL & ht t p: //hdl . handl e. net /2297/34328 \\
\hline
\end{tabular}




\section{Application and validation of a new histologic staging and grading system for primary biliary cirrhosis}

Kenichi Harada M.D. ${ }^{1)}$, Maylee Hsu M.D. ${ }^{1)}$, Hiroko Ikeda M.D. ${ }^{2)}$, Mikio Zeniya M.D. ${ }^{3)}$, and Yasuni Nakanuma M.D. ${ }^{1)}$

1) Department of Human Pathology, Kanazawa University Graduate School of Medicine, Kanazawa, Japan

2) Division of Pathology, Kanazawa University Hospital, Kanazawa, Japan

3) Gastroenterology, Jikei University Graduate School of Medicine, Tokyo, Japan

Running title: Staging and grading of primary biliary cirrhosis

Address correspondence to: Yasuni Nakanuma, MD

Department of Human Pathology

Kanazawa University Graduate School of Medicine

Kanazawa 920-8640, Japan

FAX : (0)76-234-4229 (Japan)

TEL : (0)76-265-2195 (Japan)

E-mail : pbcpsc@kenroku.kanazawa-u.ac.jp

Conflict of Interest: No conflict of interest.

Financial support: Grants-in-Aid for Scientific Research from the Ministry of Health, Labour and Welfare of Japan (The intractable hepato-biliary disease study group in Japan, 2008-2010, chief scientist Prof. Tsubouchi) and Grants-in-Aid for Scientific Research (C) from the Ministry of Education, Culture, Sports, Science, and Technology of Japan.

Abbreviation: ALP, alkaline phosphatase; ALT, alanine aminotransferase; AMA, antimitochondrial antibodies; CA, chronic cholangitis activity; CNSDC, chronic nonsuppurative destructive cholangitis; ELISA, enzyme-linked immunosorbent assay; HA, Hepatitic activity; NASH, non-alcoholic steatohepatitis; PBC, Primary biliary cirrhosis; UDCA, ursodeoxycholic acid. 


\begin{abstract}
Background:We proposed a new grading and staging system for primary biliary cirrhosis (PBC), which takes into account the degree of both chronic cholangitis activity (CA) and hepatitis activity (HA) for grading of disease activity and those of fibrosis, bile duct loss, and chronic cholestasis for staging. In this study, we validated our new system. Methods:Using liver biopsy specimens from 166 cases of PBC, chronic cholangitis with mild periductal lymphoplasmacytic infiltration including chronic nonsuppurative destructive cholangitis and combined activity of interface hepatitis and lobular hepatitis were categorized into four grades according to its degree and distribution (CA0-3 and HA0-3, respectively). For staging, because Orcein staining was not available in this study, two criteria (fibrosis and bile duct loss) were independently scored 0-3 according to their degrees and a final stage score was created from the sum. Results: Although there was a relatively uniform distribution among CA0/1/2/3, the cases of $H A 0 / 1 / 2 / 3$ were distributed as $21 \%, 64 \%, 13 \%$, and $3 \%$, respectively, with a prominence of cases categorized with none and mild hepatic activity. The distribution of stages 1-4 with our system was considerably different from that with classical system, and importantly showed a correlation with patient's outcome. Conclusions:Our system revealed that the activities of chronic cholangitis and hepatitis did not correlate with each other in degree, and that our staging system properly reflected the outcome of the PBC patients. The present study could validate the effectiveness of this new systems for characterizing the pathological condition of PBC.
\end{abstract}

Key words: primary biliary cirrhosis, staging, grading 


\section{INTRODUCTION}

Primary biliary cirrhosis (PBC) is characterized histologically by the progressive loss of intrahepatic small bile ducts, particularly interlobular bile ducts, and serologically by the presence of antimitochondrial antibodies (AMA) and the elevation of serum IgM levels. ${ }^{1,2}$ The initial lesion and the histologic hallmark of PBC is a distinctive pattern of bile duct injury referred to as chronic nonsuppurative destructive cholangitis (CNSDC). These affected bile ducts eventually disappear and chronic cholestatic features gradually develop. ${ }^{2}$ However, hepatitic changes consisting of interface hepatitis and parenchymal necroinflammatory change, which are characteristic in chronic active hepatitis, are also concurrently found in PBC and closely associated with the disease progression of PBC. ${ }^{3,4}$ Recently, in chronic liver diseases, such as chronic viral hepatitis and non-alcoholic steatohepatitis (NASH), ${ }^{5}$ grading as well as staging systems have been proposed to objectively assess the degree of disease activity. Because these pathological evaluating systems are very useful to estimate the therapeutic effect as well as to consider the therapeutic strategy, these systems are now broadly accepted and routinely used. In PBC, Bezafibrate as well as ursodeoxycholic acid (UDCA) have been used to treat biliary damage, and additional treatment with corticosteroid therapy has also been applicable for the overlapping syndrome, the hepatitic-form of PBC. Although progress has been made for the development of PBC chemotherapy, a histological evaluation of disease activity and therapeutic effect is currently subjectively performed, without a uniform pathological evaluating guideline, such as an applicable grading system for disease activity.

For evaluating the progression of PBC, classical histological staging systems, such as those proposed by Rubin et al., ${ }^{6}$ Scheuer, ${ }^{7,8}$ Popper and Schaffner, ${ }^{9}$ and Ludwig et al. ${ }^{10}$ have been used since the 1960's. However, these classical staging systems do not account for the variable histological features and/or differing stages not uncommonly seen in the same liver biopsy 
specimen or from different regions within the same liver specimen concurrently analyzed. ${ }^{3,4,11}$ Also because histological findings, particularly cholangiopathy associated with PBC, are heterogeneous in a PBC liver, the staging process itself is rather subjective and the possibility of sampling errors always should be considered in needle liver biopsies of PBC. Furthermore, important grading factors, including necroinflammatory changes of bile ducts and hepatitic activity associated with disease progression, are not fully reflected in these classical classifications. Recently, we proposed a new comprehensive grading and staging system for PBC, which takes into account the multiple histological findings of chronic cholangitis and hepatitic activity for grading and those of fibrosis, bile duct loss, and chronic cholestasis for staging. ${ }^{4}$ Furthermore, we have proposed a revised, more practical, and convenient version of this new histological staging and grading system and performed an interobserver agreement study. ${ }^{12} \underline{\text { In that study, we could }}$ obtained "fair agreement" (kappa 0.385) and concordance rate was 63.9\% for staging, though the evaluation of chronic cholangitis and hepatitic change was slight agreement (kappa 0.110 and 0.197, respectively). In this current study, we validated our new system using liver biopsy specimens of prototypic PBC and compared our analysis with our new classification system to the classical classification system.

\section{MATERIALS and METHODS}

Case selection and liver specimens: From clinicopathological data of autoimmune liver diseases previously collected from 1999 to 2000 by Prof. Zeniya, et al., ${ }^{13} 166$ cases of PBC were selected as representative of prototypical PBC. Original diagnoses of these cases were made by the on-site physicians using the standard diagnostic criteria proposed by a research group for Japanese intractable liver diseases supported by the Japanese Ministry of Health, Labor, and Welfare, ${ }^{14}$ with appropriate clinicopathological correlation: Patients whose condition meets one of the criteria 
below are diagnosed as having PBC. 1. CNSDC is histologically observed and laboratory findings do not contradict PBC, 2. AMA and/or anti-pyruvate dehydrogenase (PDH) is positive. CNSDC is not histologically observed but histological findings are compatible with PBC, and 3. Histological examination was not performed, but either AMA or anti-PDH antibody positive and clinical findings and course indicate PBC. In this study, liver biopsies were performed in all selected cases prior to treatments and the histological diagnosis of PBC was unanimously confirmed by three pathologists in all cases including AMA or PDH antibody-negative cases. From this cohort the average age was 56 years (median 52 years), with 23 males and 143 females. Laboratory data was as follows: average serum alanine aminotransferase (ALT) 64IU/L (median 50IU/L), average serum alkaline phosphatase (ALP) ratio (patient's ALP value/upper normal limit of ALP at each respective institution) 1.7 (median 1.3), and the average IgM $453 \mathrm{mg} / \mathrm{dl}$. None of the PBC patients had serological markers for HCV or HBV, and 155 patients were serologically positive for AMA by either an immunofluoresence or enzyme-linked immunosorbent assay (ELISA). Out of 23 histological findings assessed by three hepatopathologists) in our previous study, ${ }^{13}$ portal fibrosis, bile duct injury (including CNSDC and granulomatous cholangitis), bile duct loss, interface hepatitis, and lobular hepatitis were used for our classification system in this study.

Grading system and new staging system: The practical and more convenient version of our histological grading and staging systems, which is modified for needle liver biopsy in our previous study, ${ }^{12}$ are shown in Tables 1 and 2. Moreover, the representative histologies defining the grading and staging and the illustrations of Tables 1 and 2 are shown in Figs. 1 and 2, respectively. In summary, chronic cholangitis activity (CA) was categorized into 4 grades (CA0-3) according to the degree and distribution of chronic cholangitis. CA0 (no activity) was defined as absent or ambiguous bile duct damage. In CA1 (mild activity) one bile duct showed evident chronic cholangitis. In CA2 (moderate activity) two or more two bile ducts showed evident chronic cholangitis. In CA3 (marked activity) at least one damaged bile duct showed CNSDC and/or 
granulomatous cholangitis. Evident chronic cholangitis was defined as a damaged bile duct entirely surrounded by mild to moderate, duct-oriented lymphoplasmacytic inflammation. Hepatitic activity (HA) was also categorized into 4 grades (HA0-3) according to the presence and degree of interface hepatitis and lobular hepatitis. In HA0 (no activity) interface hepatitis was not present. The presence of interface hepatitis affecting at least 10 continuous hepatocytes at the interface of one portal tract or fibrous septum was categorized as HA1 (mild activity) and in two or more portal tracts or fibrous septa as HA2 (moderate activity). In HA3 (marked activity) interface hepatitis affecting at least 20 continuous hepatocytes at the limiting plate in more than half of the portal tracts or fibrous septa was present throughout the specimen, with entrapment of hepatocytes in the expanded portal tracts. While no or minimum lobular hepatitis was found in HA0, mild to moderate lobular hepatitis was found in HA1 and HA2, and moderate lobular hepatitis in HA3. Occasional zonal necrosis and bridging necrosis was regarded as HA3.

In our staging evaluation, because orcein staining was not performed in all of the cases, we used two histological criteria, fibrosis and bile duct loss. In brief the fibrosis score was calculated as follows: score 0 - almost no fibrosis was present or the fibrosis was confined only to the portal tracts; score 1- fibrosis extended to beyond the portal area, occasionally with incomplete septal fibrosis; score 2 - completely connecting septal fibrosis or bridging fibrosis with variable lobular distortion; and score 3 - established cirrhosis (extensive fibrosis with regenerative nodules). For bile duct loss, interlobular bile ducts were evaluated in well formed portal tracts with evident hepatic arterial branches and portal vein branches. ${ }^{2,15}$ Bile duct loss was scored as follows: score 0 - interlobular bile ducts were discernible in all portal tracts; score 1 - bile duct loss in less than one third of portal tracts; score 2 - bile duct loss in one third to two thirds of portal tracts; and score 3 bile duct loss in more than two thirds of portal tracts. Then the fibrosis and bile duct loss scores were combined for a total score. The total scores were categorized into the following stages: 0 stage 1 (no progression), 1-2 - stage 2 (mild progression), 3-4 - stage 3 (moderate progression), 
and 5-6 - stage 4 (advanced progression). For the classical staging system of PBC Scheuer's system was used for comparison. ${ }^{7,8}$

Prognosis survey: Approximately 10 years following the collection of the biopsies of the cohort, a follow-up survey was conducted in 2010. From the 166 patients selected in this prototypic PBC cohort, the available clinical follow-up data was obtained from 76 patients. This survey was approved by the Ethics Committee of Jikei University School of Medicine. Survival curves were calculated by the Kaplan-Meier method, and analysis was examined by the log-rank test. A p-value of less than .05 was considered to be statistically significant.

\section{RESULTS}

Distribution of activity score (grading system): The distribution of CA and HA by our grading system of the 166 PBC cases is shown in Fig.3. The percentage of cases categorized as CA0/1/2/3 was 25\%, 23\%, 27\%, and 25\%, respectively, and was relatively uniformly distributed. HA grade peaked in HA1 (mild activity), while HA3 (marked activity) was very rare. The distribution of $\mathrm{HA} 0 / 1 / 2 / 3$ was $21 \%, 64 \%, 13 \%$, and $3 \%$, respectively.

Distribution of stages and comparison with the classical system: The distribution of stages in our staging systems using two histological criteria (fibrosis and bile duct loss) and in the classical system by Scheuer ${ }^{7,8}$ is shown in Fig.4. In our system, stage 1/2/3/4 was distributed as 21\%, 45\%, 21\%, and 13\%, respectively, with stage 2 (mild progression) being the most frequent. In comparison to the classical classification, in our system stage 1 cases were markedly decreased and other stages were mildly increased. In particular, from the 46 stage 1 cases as defined by the classical staging system, 32 cases with no fibrosis (fibrosis score 0) and mild bile duct loss (bile duct loss score 1) and 21 cases with mild fibrosis (fibrosis score 1) and no bile duct loss (bile duct score 0 ) were estimated as stage 2 (total stage score of 1 ) in our new staging system. Additionally, 
10 cases, from the classical classification stage 3, showing non-cirrhosis (fibrosis score 2) and marked bile duct loss (bile duct loss score 3) were moved to stage 4 in the new system. Fig.5 shows the distribution of CA and HA in each stage. Cases of stages 1, 2, and 3 were scattered amongst the entire spectrum of CA and HA grades, while all cases of stage 4, except for one case, belonged to CA0 or CA1.

Prognostic value of the new staging system: Out of the 76 patients with available outcome data, five patients underwent liver transplantation and three died from liver failure or hepatocellular carcinoma (Table 3). Five cases were lost to follow-up. The remaining patients were confirmed as alive as of 2010. By the Kaplan-Meier estimator, the overall survival curve for the 76 PBC patients is shown in Fig.6. Although the distribution of staging using the classical system could not divide the survival ratio, the survival curve by our system created a statistically significant difference in the survival ratio among stages (the log-rank test, $\mathrm{p}=0.02$ ). The 5-year survival probabilities for stage $1 / 2 / 3 / 4$ patients were $100 \%, 100 \%, 100 \%$, and $80 \%$, and the 10-year ones were $100 \%$, 92\%, 82\%, and 53\%, respectively.

\section{DISCUSSION}

Over the last few decades the pathogenesis and histogenesis of PBC have been gradually clarified, and there has been growing evidence for the clinical benefits of treatment with UDCA and Bezafibrate and its combination with corticosteroid for PBC-AIH overlap syndrome. ${ }^{16-18}$ With these recent advances and developments, pathological assessment for therapeutic effect is greatly needed to guide and evaluate PBC treatment, however an objective and unified evaluation system with clearly outlined criteria has not been developed. Chronic cholangitis, including CNSDC and bile duct loss, is thought to be a histological hallmark of PBC and useful for the pathological diagnosis of PBC. But hepatitic changes, such as interface hepatitis and lobular hepatitis, are also 
not uncommonly found in the histology of PBC, suggesting that hepatitic changes may also play a role in the histogenesis and disease progression of PBC. ${ }^{2,3}$ Therefore, we have proposed a new grading system in PBC which separately evaluates chronic cholangitis (CA) and hepatitis (HA). ${ }^{4,12}$ This independent evaluation is necessary for a more accurate and precise analysis of the pathogenesis of PBC and the assessment of the effect of various therapies.

In the present study, we evaluated 166 cases of clinicopathologically correlated prototypic PBC using our grading system. Following analysis, we showed that the degree of chronic cholangitis (CA0-3) was nearly uniformly distributed. Although chronic cholangitis is a distinguishing feature of $\mathrm{PBC}$, this finding suggests that various degrees of chronic cholangitis are found in PBC and up to 25\% of PBC cases may not show cholangitis in a given biopsy specimen. However, because grades of CA0 were also found in stage 4 cases showing complete bile duct loss (Fig.5), the significance of evaluating the chronic cholangitis activity in these types of cases should be deliberated. In contrast to cholangitis activity, a distribution of hepatitic activity deviated to the mild category (HA1) in prototypic PBC. In addition to bile duct lesions, hepatitic activity, consisting of interface hepatitis and/or lobular hepatitis, are concurrently found in PBC, but their degrees are usually mild, compared with those found in chronic viral hepatitis and autoimmune hepatitis (AIH). Therefore, the present data, demonstrating that most of the PBC cases were of HA0 or HA1, is in accordance with the typical findings of PBC. Moreover, our results revealed that PBC cases showing marked hepatitic activity (HA3) only occurred in 3\% of PBC cases. PBC and AIH are both autoimmune diseases, which selectively affect cholangiocytes and hepatocytes, respectively, but PBC-AIH overlap or the hepatitic-form of PBC also has been recognized. ${ }^{17,18}$ Because our grading system can independently evaluate cholangitis and hepatitis, this system is very useful in evaluating the differential diagnosis for these diseases, including a possible overlap syndrome, and in also guiding therapeutic strategy. For example, in PBC-AIH overlap syndrome, the HA score has been reported to be significantly higher than the CA score. ${ }^{19}$ 
In the majority of chronic liver diseases such as chronic viral hepatitis, the degree of fibrosis constitutes the basis for staging. ${ }^{5,20,21}$ However, because cholestasis, in addition to fibrosis, is also a major causative factor of liver failure in PBC, ${ }^{3,4,22,23}$ liver transplantation cases of non-cirrhotic livers (Stage 3 in the classical classification) showing terminal liver failure have often been encountered. Therefore, we have proposed our new staging system, which takes into consideration multiple histologic features: fibrosis, bile duct loss, and if orcein staining is available deposition of copper-binding proteins reflecting chronic cholestasis. ${ }^{4,12}$ Using these multiple histological features for staging, we could more precisely define the stage of PBC and reduce sampling errors in the histological evaluation of PBC. The present study using our system revealed that prototypic PBC cases were distributed as follows: stage 1 (22\%), stage 2 (45\%), stage 3 (22\%), and stage 4 (12\%), with stage 2 (mild progression) being the most frequent. Compared to the classical classification, there was a significant difference in the distribution of stages. Particularly, PBC cases with no or mild fibrosis (fibrosis score 0 or 1 ) and mild bile duct loss (bile duct score 1 ) were transferred from stage 1 in the classical system to stage 2 in our new staging system. Also, the possible establishment of a new category of a 'no progression state' (stage 1) is unique to our system. Since the number of stage 1 cases in our system was small, the significance of this category should be analyzed. Several concepts such as a pre-symptomatic state are raised as possibilities, but a more detailed follow-up study is needed to clarify its significance. Moreover, the number of stage 4 cases was increased in our new system, as compared with the classical system. The reason for this change is that the non-cirrhotic cases (fibrosis score 2) with marked bile duct loss (bile duct score 3) were transferred from stage 3 (non-cirrhotic stage) in the classical classification to stage 4 in our new system. Because these transferred cases already had severe liver dysfunction clinically and the score of bile duct loss correlated with high serum levels of ALP, these cases should be categorized as the terminal stage (Stage 4$){ }^{23}$ In contrast to the classical system, the survival curve by our system created a significant difference in survival ratios among 
stages, suggesting that our new staging system precisely reflects the progression of PBC patients.

In this study, we evaluated the application and validation of a new histologic staging and grading system using liver biopsy specimens of prototypic PBC patients. Our grading system is unique in that it separately evaluates chronic cholangitis activity (CA) and hepatitic activity (HA), and our results revealed that the activities of chronic cholangitis and hepatitis do not correlate in degree. The degree of chronic cholangitis (CA0-3) was nearly equally distributed, but the majority of cases had HA0 or HA1 in prototypic PBC. Staging by our new system also differed with the staging by the classical system and importantly correlated with the outcome of PBC patients. Our new system is slightly complicate and it may be a little burdensome for pathologists. Moreover, this system does not reflect the distinguishing condition of PBC patients showing marked portal hypertension (nodular regenerative hyperplasia histology) and acute cholestasis, which may reflect patients' poor outcome. However, we believe that this system will provide more objective information from liver biopsy specimens of PBC to clinicians. In conclusion, our new grading and staging system is a very effective and comprehensive system for evaluating the activity, progression, outcome, and treatment effect in PBC, and also can be used for studying and diagnosing autoimmune liver diseases including PBC and PBC/AIH overlap syndrome.

\section{ACKNOWLEDGEMENTS}

This work was supported by Grants-in-Aid for Scientific Research from the Ministry of Health, Labour and Welfare of Japan (The intractable hepato-biliary disease study group in Japan, 2008-2010, chief scientist Prof. Tsubouchi) and Grants-in-Aid for Scientific Research (C) from the Ministry of Education, Culture, Sports, Science, and Technology of Japan. Moreover, the authors are grateful to members of the study group who participated in this study and to Kumiko Isse, M.D. (Department of Pathology, Division of Transplantation, University of Pittsburgh 
Harada et al, - 12 -

Medical Center) for drawing up nice figures. 


\section{REFERENCES}

1. Kaplan MM. Primary biliary cirrhosis. N Engl J Med 1996; 335: 1570-1580.

2. Nakanuma Y, Ohta G. Histometric and serial section observations of the intrahepatic bile ducts in primary biliary cirrhosis. Gastroenterology 1979; 76: 1326-1332.

3. Nakanuma Y, Saito K, Unoura M. Semiquantitative assessment of cholestasis and lymphocytic piecemeal necrosis in primary biliary cirrhosis: a histologic and immunohistochemical study. J Clin Gastroenterol 1990; 12: 357-362.

4. Hiramatsu K, Aoyama H, Zen Y, et al. Proposal of a new staging and grading system of the liver for primary biliary cirrhosis. Histopathology 2006; 49: 466-478.

5. Brunt EM, Janney CG, Di Bisceglie AM, et al. Nonalcoholic steatohepatitis: a proposal for grading and staging the histological lesions. Am J Gastroenterol 1999; 94: 2467-2474.

6. Rubin E, Schaffner F, Popper H. Primary Biliary Cirrhosis. Chronic Non-Suppurative Destructive Cholangitis. Am J Pathol 1965; 46: 387-407.

7. Scheuer P. Primary biliary cirrhosis. Proc R Soc Med 1967; 60: 1257-1260.

8. Scheuer P, Lefkowitch JH, editors. Liver biopsy interpretation. 7th ed. Philadelphia: Elsevier Saunders Ltd; 2007.

9. Popper H, Schaffner F. Nonsuppurative destructive chronic cholangitis and chronic hepatitis. New York: Grune \& Stratton, Inc,, 1970: 336-354.

10. Ludwig J, Dickson ER, McDonald GS. Staging of chronic nonsuppurative destructive cholangitis (syndrome of primary biliary cirrhosis). Virchows Arch A Pathol Anat Histol 1978; 379: 103-112.

11. Scheuer PJ. Ludwig Symposium on biliary disorders--part II. Pathologic features and evolution of primary biliary cirrhosis and primary sclerosing cholangitis. Mayo Clin Proc 1998; 73: 179-183.

12. Nakanuma Y, Zen Y, Harada K, et al. Application of a new histological staging and grading system for primary biliary cirrhosis to liver biopsy specimens: Interobserver agreement. Pathol Int 2010; 60: 167-174.

13. Zeniya M, Watanabe F, Morizane T, et al. Diagnosing clinical subsets of autoimmune liver diseases based on a multivariable model. J Gastroenterol 2005; 40: 1148-1154.

14. Sasaki $\mathrm{H}$, Inoue $\mathrm{K}$, Higuchi $\mathrm{K}$, et al. Primary biliary cirrhosis in Japan: national survey by the Subcommittee on Autoimmune hepatitis. Gastroenterol Jpn 1985; 20: 476-485.

15. Crawford AR, Lin XZ, Crawford JM. The normal adult human liver biopsy: a quantitative reference standard. Hepatology 1998; 28: 323-331.

16. Kita R, Takamatsu S, Kimura T, et al. Bezafibrate may attenuate biliary damage associated with chronic liver diseases accompanied by high serum biliary enzyme levels. J Gastroenterol 2006; 41: 686-692.

17. Yokokawa J, Saito H, Kanno Y, et al. Overlap of primary biliary cirrhosis and autoimmune hepatitis: Characteristics, therapy, and long term outcomes. J Gastroenterol Hepatol 2010; 25: 376-382.

18. Boberg KM, Chapman RW, Hirschfield GM, et al. Overlap syndromes: the International Autoimmune 
Hepatitis Group (IAIHG) position statement on a controversial issue. J Hepatol 2011; 54: 374-385.

19. Tanaka A, Harada K, Ebinuma H, et al. Primary biliary cirrhosis - Autoimmune hepatitis overlap syndrome: A rationale for corticosteroids use based on a nation-wide retrospective study in Japan. Hepatol Res 2011; 41: 877-886.

20. Desmet VJ, Gerber M, Hoofnagle JH, et al. Classification of chronic hepatitis: diagnosis, grading and staging. Hepatology 1994; 19: 1513-1520.

21. Ishak K, Baptista A, Bianchi L, et al. Histological grading and staging of chronic hepatitis. $J$ Hepatol 1995; 22: 696-699.

22. Portmann B, Popper H, Neuberger J, et al. Sequential and diagnostic features in primary biliary cirrhosis based on serial histologic study in 209 patients. Gastroenterology 1985; 88: 1777-1790.

23. Ricci P, Therneau TM, Malinchoc M, et al. A prognostic model for the outcome of liver transplantation in patients with cholestatic liver disease. Hepatology 1997; 25: 672-677. 


\section{FIUGRE LEGENDS}

Fig.1 Representative findings defining grading and staging of primary biliary cirrhosis. A: Chronic non-suppurative destructive cholangitis (arrow). Chronic cholangitis activity 3 (CA3). HE staining. B: Evident chronic cholangitis with moderate periductal lymphocytic infiltration (arrow). Part of the limiting plate shows interface hepatitis affecting approximately 10 hepatocytes (arrowheads). HE staining. C: Well-formed interlobular bile ducts are lost in portal tracts (arrows). Interface hepatitis affecting approximately 20 hepatocytes at the interface (arrowheads). Hepatitic activity 3 (HA3). HE staining. D: In parenchyma, moderate lobular hepatitis consisting of several focal necrosis is found (arrows). HE staining. E: Portal tract showing fibrous enlargement without fibrous septa formation (Score 1 of fibrosis). Reticulin staining. F: Deposition of granules in many hepatocytes are found around enlarged portal tract. Score 3. Orcein staining.

Fig.2 Illustration of Tables 1 and 2. A: Grading of chronic cholangitis and hepatitic activity in primary biliary cirrhosis. B: Scoring for stage of primary biliary cirrhosis.

Fig 3. The distribution of chronic cholangitis activity (CA) and hepatitis activity (HA) by our new grading system using the prototypic 166 PBC cases. (A): The percentage of cases categorized as CA0, CA1, CA2 and CA3 was 25\%, 23\%, 27\%, and 25\%, respectively. (B): The distribution of cases as HA0, HA1, HA2, and HA3 was 21\%, 64\%, 13\%, and 3\%, respectively.

Fig 4. The distribution of stages in our new staging systems using two histological criteria (fibrosis and bile duct loss) and in the classical system by Scheuer. In our system, stages $1,2,3$, and 4 were $21 \%, 45 \%, 21 \%$, and $13 \%$, respectively. In contrast, stages $1,2,3$, and 
4 were 59\%, 25\%, 10\%, and 5\%, respectively, by the classical system.

Fig 5. Distribution of chronic cholangitis activity (CA) and hepatitis activity (HA) in each stage. Cases of stages 1, 2, and 3 were widely scattered among all grades of CA and HA, but all cases of stage 4, except one case (CA2, HA1), belonged to CA0 or CA1.

Fig.6. Kaplan-Meier survival analysis based on stages 1-4 using the outcome from 76 PBC patients. (A): The survival curve estimated by our new staging system created a statistically significant difference in survival ratio among stages (the log-rank test, $\mathrm{p}=0.02)$. (B): In the survival curve estimated by the classical system, there was a reversal between stage 1 and stage 2 (the log-rank test, $\mathrm{p}=5.87$ ). Parentheses denote number of cases. 
Harada et al, - 1 -

Table 1. Grading of chronic cholangitis and hepatitic activity in primary biliary cirrhosis

Chronic cholangitis activity (CA)

\begin{tabular}{ll} 
CA0 (no activity) & No cholangitis, but mild duct epithelial damage may be present \\
CA1 (mild activity) & 1 bile duct with evident chronic cholangitis \\
CA2 (moderate activity) & $>2$ bile ducts with evident chronic cholangitis \\
CA3 (marked activity) & $>1$ bile duct with CNSDC \\
\hline
\end{tabular}

(CNSDC: chronic nonsuppurative destructive cholangitis.)

Hepatitic activity (HA)

\begin{tabular}{ll} 
HA0 (no activity) & $\begin{array}{l}\text { No interface hepatitis, and no or minimum lobular hepatitis } \\
\text { HA1 (mild activity) }\end{array}$ \\
& $\begin{array}{l}\text { Interface hepatitis affecting }>10 \text { continuous hepatocytes in } 1 \text { portal tract or fibrous septum } \\
\text { and mild to moderate lobular hepatitis }\end{array}$ \\
HA2 (moderate activity) & $\begin{array}{l}\text { Interface hepatitis affecting }>10 \text { continuous hepatocytes in }>2 \text { portal tracts or fibrous septa, } \\
\text { and mild to moderate lobular hepatitis }\end{array}$ \\
HA3 (marked activity) & Interface hepatitis affecting $>20$ continuous hepatocytes in $>1 / 2$ of portal tracts, \\
& and moderate lobular hepatitis or bridging or zonal necrosis \\
\hline
\end{tabular}


Harada et al, - 2 -

Table 2. Scoring for stage of primary biliary cirrhosis

Scoring of fibrosis

Score $0 \quad$ No portal fibrosis, or fibrosis limited to portal tracts

Score 1 Portal fibrosis with periportal fibrosis or incomplete septal fibrosis

Score 2 Bridging fibrosis with variable lobular disarray

Score 3 Liver cirrhosis with regenerative nodules and extensive fibrosis

\section{Scoring of bile duct loss}

\begin{tabular}{llll} 
Score & $\mathbf{0}$ & No bile duct loss \\
Score & 1 & Bile duct loss in $<1 / 3$ of portal tracts \\
Score & 2 & Bile duct loss in $1 / 3$ to $2 / 3$ of portal tracts \\
Score & 3 & Bile duct loss in $>2 / 3$ of portal tracts \\
\hline
\end{tabular}

Scoring of deposition of orcein-positive granules

\begin{tabular}{llll}
\hline Score & 0 & No deposition of granules \\
Score & 1 & Deposition of granules in a few periportal hepatocytes in $<1 / 3$ of portal tracts \\
Score & 2 & Deposition of granules in several periportal hepatocytes in $1 / 3$ to $2 / 3$ of portal tracts \\
Score & 3 & Deposition of granules in many hepatocytes in $>2 / 3$ of portal tracts \\
\hline
\end{tabular}

Staging by sum total of three and two criteria

\begin{tabular}{lcc}
\hline & \multicolumn{2}{c}{ Sum of score } \\
\cline { 2 - 3 } Stage & 3 criteria & 2 criteria \\
\hline Stage 1 (no progression) & 0 & 0 \\
Stage 2 (mild progression) & $1-3$ & $1-2$ \\
Stage 3 (moderate progression) & $4-6$ & $3-4$ \\
Stage 4 (advanced progression) & $7-9$ & $5-6$ \\
\hline
\end{tabular}

3 criteria: fibrosis, bile duct loss and deposition of orcein-positive granules. 2 criteria: fibrosis and bile duct loss 
Harada et al, - 3 -

Table 3. List of fatal or liver transplant cases

\begin{tabular}{|c|c|c|c|c|c|c|}
\hline \multirow[b]{2}{*}{ Sex } & \multirow[b]{2}{*}{ Age } & \multirow[b]{2}{*}{$\begin{array}{c}\text { CA } \\
\text { score }\end{array}$} & \multirow[b]{2}{*}{$\begin{array}{l}\text { HA } \\
\text { score }\end{array}$} & \multicolumn{2}{|c|}{ Histological staging } & \multirow[b]{2}{*}{ Outcome } \\
\hline & & & & $\begin{array}{c}\text { New } \\
\text { classification }\end{array}$ & $\begin{array}{l}\text { Scheuer's } \\
\text { classification }\end{array}$ & \\
\hline Male & 73 & 2 & $\mathbf{0}$ & Stg 2 & Stg 1 & die from HCC 10 years later (83yrs) \\
\hline Female & 36 & 3 & $\mathbf{0}$ & Stg 2 & Stg 1 & undergo liver transplantation 6 years later (42yrs) \\
\hline Female & 46 & 2 & 1 & Stg 2 & Stg 1 & undergo liver transplantation 6 years later (52yrs) \\
\hline Female & 56 & 1 & 1 & Stg 3 & Stg 2 & undergo liver transplantation 10 years later (66yrs) \\
\hline Female & 34 & 2 & 1 & Stg 3 & Stg 3 & undergo liver transplantation 6 years later (40yrs) \\
\hline Female & 63 & 2 & 3 & Stg 3 & Stg 3 & die from liver failure 8 years later (71yrs) \\
\hline Female & 50 & 1 & 1 & Stg 4 & Stg 4 & die from liver failure 1 year later (51yrs) \\
\hline Male & 39 & 3 & 2 & Stg 4 & Stg 4 & undergo liver transplantation 7 years later (46yrs) \\
\hline
\end{tabular}

